\title{
Polymorphonuclear leucocyte traffic in lung inflammation
}

The margination of polymorphonuclear leucocytes (PMNs) in microvessels and their migration out of the vascular space is a multistep process that is regulated by a cascade of molecular events involving several families of adhesion proteins. Adams and Shaw ${ }^{1}$ have recently reviewed the evidence for the hypothesis that this process begins with a selectin-mediated rolling of the PMNs on the endothelial surface of the post-capillary venules followed by a chemokine-induced triggering of a stronger, integrinmediated adherence between the leucocyte and the endothelial surface that occurs in preparation for migration. The data that support this hypothesis are impressive, particularly for the systemic vessels where both margination and migration apparently occur in post-capillary venules. However, in the pulmonary circulation there are compelling reasons to believe that the circulating cells slow down, adhere, and migrate through the endothelium of the capillaries, and that only a fraction of the PMNs passing through an inflammatory site become significantly activated to migrate out of the vascular space. ${ }^{2}$

The progressive activation of PMNs is most easily investigated in vitro. Studies using a prototypic agonist of neutrophils, f-met-leu-phe (FMLP), have shown that low concentrations $\left(10^{-12}-10^{-10} \mathrm{M}\right)$ are associated with reorganisation of the cytoskeleton to a configuration which allows the cells to move independently. ${ }^{3-5}$ As the FMLP concentration is increased, the specific granules release CD $11 \mathrm{~b} / \mathrm{CD} 18$ onto the surface and L-selectin is shed. ${ }^{6}$ These events occur in association with increased intracellular levels of inositol trisphosphate and calcium. ${ }^{7}$ However, it is only at very high concentrations $\left(10^{-7}-10^{-6} \mathrm{M}\right)$ that the major cytotoxic responses of azurophilic granule release and superoxide production are demonstrable. ${ }^{6}$ Other agonists that use structurally similar receptors (interleukin 8, platelet aggregating factor, $\mathrm{C5a}$ ) have similar dose-dependent responses but these agonists can also prime the cell and amplify its cytotoxic response. ${ }^{89}$ Priming refers to an enhanced response that results from the sequential addition of priming agent and agonist. ${ }^{9}$ Priming of the superoxide response usually occurs at intermediate doses sufficient to cause increases in intracellular calcium and specific granule release, but just at or below the doses necessary to release superoxide. ${ }^{89}$ Other mediators such as the cytokines (interleukin 1, granulolytemacrophage colony stimulating factor, tumour necrosis factor) and endotoxin are incapable of producing a full repertoire of neutrophil responses.

The relationship between the graded neutrophil response in vitro and their behaviour in vivo has only been partially determined. Their response within the vascular space where agonist concentrations are low is probably limited to the assembly of actin, a reduction in cell deformability, upregulation of $C D 11 \mathrm{~b} / \mathrm{CD} 18$, and a loss of $\mathrm{L}$-selectin from the cell surface. Once adherent to the endothelium, the cells move along chemotactic or haptotactic gradients centred at the site of injury. This allows the PMNs to encounter increasing agonist concentrations that first prime and then trigger a full cytotoxic response. Any abnormality in this sequence that allows large numbers of PMNs to become fully activated before leaving the vascular space puts the lung tissue itself at risk of injury.

Approximately 8640 litres of blood pass through the pulmonary circulation of an adult human in 24 hours. As each litre of blood contains $4.5 \times 10^{9}$ PMNs about
$3.8 \times 10^{13}$ PMNs pass through the pulmonary microvessels each day. The human PMN has a similar diameter to, but larger volume than, the erythrocytes, ${ }^{10}$ and the pulmonary capillary bed is formed by an interconnecting network of $10^{11}$ short segments with an average diameter of $7 \cdot 5(2 \cdot 3)$ $\mu \mathrm{m}$ and an average length of $14.4(5 \cdot 8) \mu \mathrm{m} .{ }^{10-12}$ The distributions of PMN diameters $(6.8(0.8) \mu \mathrm{m})$ overlap that of the capillary segments by approximately $38 \%$, which means that circulating cells encounter segments that restrict their passage on each transit through the lung. ${ }^{12}$ During this encounter the PMNs deform and come into close contact with the capillary endothelial surface. ${ }^{12}$ Because the post-capillary venules have much larger diameters and a total endothelial surface area of only five square metres compared with the $>100$ square metres available in capillaries, there is a much greater opportunity for interactions to occur between PMNs and the endothelium in the capillaries than in the post-capillary venules of the lung. ${ }^{13}$

Direct observations of the pleural surface ${ }^{14}$ and indirect measurements from deeper lung regions ${ }^{11}$ have shown that it takes leucocytes much longer than the erythrocytes to travel through capillaries. The slower movement of the PMNs compared with the erythrocytes is a result of the fact that they are about 300 times less deformable than erythrocytes and are held up for longer periods in narrow segments. ${ }^{1215}$ The interconnecting network of segments that forms the capillary bed allows the erythrocytes to have median transit times of one second compared with 120 seconds for PMNs. ${ }^{11}$

The concentration of PMNs with respect to erythrocytes that results from this difference in speed can be influenced by mechanical events affecting either the vessels or the circulating cells. Studies of patients undergoing simultaneous right and left cardiac catheterisation have shown that an immediate arteriovenous difference for PMNs developed when the lung capillaries were compressed by raising alveolar pressure and shifting lung from zone III to zone II conditions. ${ }^{16}$ Animal studies subsequently showed that the arteriovenous difference produced in this way disappears with time as a new equilibrium is established at the longer PMN transit times. ${ }^{17}$ PMNs rapidly accumulate in the lung when stimulated by the infusion of zymosan-activated plasma. ${ }^{18}$ This accumulation is a twostep process where reduced PMN deformability dominates the initial retention and CD11/CD18-mediated adherence plays a secondary role. ${ }^{19}$ Interestingly, the massive PMN margination produced by the infusion of activated plasma causes only minor changes in the epithelial permeability with no increase in extravascular lung water or protein. ${ }^{20}$ This suggests that the plasma-derived stimuli that massively increase PMN margination fail to activate the cells to a point where they are capable of injuring lung tissue.

Downey et $a l^{21}$ showed that in the lung PMNs migrate out of capillaries rather than post-capillary venules and Doerschuk et $a l^{22}$ showed that this migration could be CD18 independent in the lung but not in the systemic circulation. ${ }^{21}$ Interestingly, only $1-2 \%$ of the cells delivered to an area of pneumonia actually migrate out into the airspaces ${ }^{2}$ compared with the $60-80 \%$ migration observed in vitro. ${ }^{23}$ If both lungs of an adult human weigh $1000 \mathrm{~g}$ and have a total air capacity of seven litres, the maximum available airspace would be $7 \mathrm{ml} / \mathrm{g}$. If the entire lung receives about $10^{13}$ leucocytes in 24 hours, each gram will 
receive $10^{10}$ cells and a $1 \%$ migration of the delivered cells - that is, $10^{8}$ would occupy a volume equal to all of the available airspace. This suggests that PMN migration into inflammatory sites in human lungs must be closer to the $1 \%$ of delivered cells observed in vivo than the much higher values demonstrated in vivo.

In local streptococcal infections in rabbit lungs PMN migration into airspaces was limited to the site of inoculation, whereas PMN margination was increased throughout both lungs. ${ }^{2}$ The partial activation of the PMNs responsible for this widespread margination in lung capillaries in the early stages of pneumonia is similar to the margination induced by the infusion of activated plasma ${ }^{20}$ in that it causes little damage. However, full activation of this large intravascular pool of cells by a subsequent bacteraemia may result in complete PMN activation with the widespread destruction of the lung surface.

In summary, the current paradigms for migration of PMNs out of post-capillary venules in systemic vessels cannot be directly applied to the lung. The size of the PMNs relative to the microvascular segments ensures that there will always be close apposition of PMNs to the endothelium as they transit through lung capillaries. Their relatively slow speed in the pulmonary capillaries provides ample opportunities for contact between the PMNs and endothelium. The contact can be widespread and prolonged by the presence of inflammatory stimuli and may contribute to the increased risk of widespread lung injury associated with bacteraemia in pneumococcal infections. ${ }^{24}$

Reprint requests to Professor J C Hogg

University of British Columbia Pulmonary

JAMES C HOGG Research Laboratory,

St Paul's Hospital,

1081 Burrard Street,

Vancouver, B.C.

V6Z 1 Y6 Canada

1 Adams DH, Shaw S. Leukocyte-endothelial interaction and regulation of leukocyte migration. Lancet 1994;343:831-6.

2 Doerschuk CM, Markos J, Coxson HO, English D, Hogg JC. Quantitation of neutrophil migration in acute bacterial pneumonia in rabbits. $\mathcal{F} A p p l$ Physiol 1994;77:2593-9.
3 Casale TB, Abbas MK, Carolan EJ. Degree of neutrophil chemotaxis is dependent upon the chemoattractant and barrier. Am $\mathcal{f}$ Respir Cell Mol Biol 1992;7:112-7.

$4 \mathrm{Al}-$ Mohanna FA, Hallett MB. Actin polymerization in neutrophils is triggered without a requirement for a rise in cytoplasmic $\mathrm{Ca}^{2+}$. Biochem ₹ 1990;266:669-74

5 Dewald B, Baggiolini M. Activation of NADPH oxidase in human neutrophils. Synergism between AMLP and the neutrophil products PAF and LTB4. Biochem Biophys Res Commun 1985;128:297-304.

6 Petrequin PR, Todd RF III, Devall LJ, Boxer LA, Curnette JT III. Association between gelatinase release and increased plasma membrane expression of the Mol glycoprotein. Blood 1987;69:605-10.

7 Walker BA, Hagenlocker BE, Stubbs EB Jr, Sandborg RR, Agranoff BW, Ward PA. Signal transduction events and Fc gamma R engagement in human neutrophils stimulated with immune complexes. F Immunol 1991; human neutropl

8 Gay JC, Beckman JK, Zaboy KA, Lukens JN. Modulation of neutrophil oxidative responses to soluble stimuli by platelet-activating factor. Blood 1986;67:931-6

9 Walker BA, Ward PA. Priming and signal transduction in neutrophils. Biol Signals 1992;1:237-49.

10 Hogg JC. Neutrophil kinetics in lung injury. Physiol Rev 1987;67:1249-95.

11 Hogg JC, Coxson HO, Brumwell M-L, Beyers N, Doerschuk CM, MacNee W, et al. Erythrocyte and polymorphonuclear cell transit time and concentration in human pulmonary capillaries. $\mathcal{F}$ Appl Physiol 1994;77:1795800 .

12 Doerschuk CM, Beyers N, Coxson HO, Wiggs B, Hogg JC. Comparison of neutrophil and capillary diameters and their relation to neutrophil sequestration in the lung. $\mathcal{F}$ Appl Physiol 1993;74:3040-5.

13 Staub NC. Pulmonary edema. Physiol Rev 1974:54:678-811.

14 Lien DC, Wagner WW Jr, Capen RL, Haslett C, Hanson WL, Hoffmeister $\mathrm{SE}$, et al. Physiological neutrophil sequestration in the lung: visual evidence for location in capillaries. $\mathcal{F}$ Appl Physiol 1987;62:1236-43.

15 Chien S. Role of blood cells in microcirculatory regulation. Eugene M. Landis Award. Microvasc Res 1985;29:129-51.

16 Markos J, Hopper RO, Kavanagh-Gray D, Wiggs BR, Hogg JC. Effect of raised alveolar pressure on leukocyte retention in the human lung. $\mathcal{F} A p p l$ Physiol 1990;69:214-21.

17 Markos J, Doerschuk CM, English D, Wiggs BR, Hogg JR. Effect of positive and expiratory pressure on leukocyte transit in rabbits. $\mathcal{f}$ Appl Physiol 1993;74:2627-33.

18 Doerschuk CM, Allard MF, Hogg JC. Neutrophil kinetics in rabbits during infusion of zymosan-activated plasma. $\mathcal{F}$ Appl Physiol 1989;67:88-95.

19 Doerschuk CM. The role of CD18-mediated adhesion in neutrophil sequestration induced by infusion of activated plasma. Am $\mathcal{F}$ Respir Cell Mol Biol 1992;1:140-5.

20 Gie RP, Doerschuk CM, English D, Coxson HO, Hogg JC. Neutrophil associated lung injury after the infusion of zymozan-activated plasma. $尹$ Appl Physiol 1991;70:2471-8.

21 Downey GP, Worthen GS, Henson PM, Hyde DM. Neutrophil sequestration and migration in localized pulmonary inflammation. Am Rev Respir Dis 1993;147:168-76.

22 Doerschuk CM, Win RK, Coxson HO, Harlan JM. CD18-dependent and independent mechanisms of neutrophil adherence in the pulmonary and independent mechanisms of neutrophl adherence in the

23 Smith CW, Marlin SD, Rothlein R, Toman C, Andersen DC. Cooperative interactions of LFA1 and Macl with intercellular adhesion molecule 1 in facilitating adherence and transendothelial migration of human neutrophils facilitating adherence and transendotheli

24 Austrian R, Gold R. Pneumococcal bacteremia with special reference to bacteremic pneumococcal pneumonia. Ann Intern Med 1964;60:759-76. 